\title{
Política y presupuesto de gasto social: ¿Han tenido efecto las políticas inclusivas del actual gobierno?
}

\author{
Enrique Samanamud \\ Universidad de Lima \\ Recibido: 11/08/2015 / Aprobado: 03/12/2015
}

\begin{abstract}
Resumen: El documento ${ }^{1}$ es un breve análisis de la política social llevada a cabo por el actual gobierno desde julio de 2011. Según versión de sus principales representantes, intenta darle un nuevo enfoque al gasto social, apuntando al problema social de la pobreza ligado a la exclusión social, tomando como base no solo la reducción promedio de la pobreza, sino principalmente la reducción de las disparidades sociales. Los resultados preliminares en este sentido son aún ambiguos, aunque alentadores con respecto a las metas trazadas por el propio gobierno, reconociendo la nueva etapa del ciclo de crecimiento que atraviesa nuestra economía.
\end{abstract}

Palabras clave: política social / gasto social / exclusión / pobreza / disparidades sociales.

\section{Political and Social Spending Budget: Have Had Effect Inclusive Policies of the Current Government?}

АвsтRAст: This paper is a brief analysis of the social policy strategy pursued by the current Government since July 2011; which according to its leading figures attempts to set a new approach to social spending focusing on poverty as a social problem related to social exclusion, not only based on the average poverty reduction, but also and mainly on the reduction of social disparities. Preliminary results in this regard are still ambiguous, although positive with respect to the goals set by the current Government in terms of social policy, recognizing the new phase of growth that our Peruvian economy is experiencing

Keywords: social policy / social spending / exclusion / poverty / social disparities

1 Para el resumen de este documento se contó con la colaboración del alumno Diego Palomino Bogdanovich, de la Facultad de Economía. 


\section{INTRODUCCIÓN}

Esta investigación ${ }^{2}$ tiene como objetivo hacer una evaluación preliminar de la política social llevada a cabo por el actual gobierno y destacar que, aunque resulte imprescindible contar con una política social, el elemento central que resuelve la pobreza es el crecimiento. El análisis se inicia identificando los diversos enfoques sobre el problema de la pobreza. Toma como punto de partida la teoría del capital social, propuesta por autores como Bordieu, Coleman o Putnam, entre otros. Incluye también el enfoque de capacidades y derechos de Amartya Sen, el enfoque de exclusión social nacido en Francia a finales de los años setenta y difundido por la Organización Internacional del Trabajo, y finalmente el enfoque estructural de la pobreza como resultado de los patrones de desigualdad y de las estructuras de poder imperantes.

En el segundo capítulo se realiza una conceptualización y clasificación de la política social como instrumento para reducir la pobreza, resaltando los cambios de enfoque surgidos en este tema en las últimas décadas. Posteriormente se establece la evolución de la política social en el Perú, de acuerdo a los diferentes enfoques analizados sobre la pobreza.

De igual forma, en el tercer capítulo se evalúa el gasto social realizado en los últimos 25 años, haciendo comparaciones por periodo de gobierno.

En el cuarto capítulo se define la política de gasto social del actual gobierno bajo el enfoque de la inclusión social. Se analiza la reducción promedio de la pobreza, siguiendo la premisa de las disparidades sociales. Ello implica atacar la desigualdad como factor que contribuye a perennizar la pobreza, debido a que se manifiesta en las enormes disparidades entre el ámbito rural y el urbano, entre la sierra y selva versus la costa, etc. Ello ha demandado un cambio en el análisis de las causas de la pobreza, y transitar hacia una política de ciclo de vida que pueda enfrentar dichas disparidades trasversalmente. Además, se efectúa una evaluación a partir del análisis preliminar de algunos indicadores, comparando su avance a la fecha con respecto a las propias metas que estableció este gobierno al 2016.

2 El presente documento es un breve resumen actualizado de la tesis de 2014 para optar por el grado de Master en Administración Financiera y Hacienda Pública, otorgado al autor por la Universidad Nacional de Educación a Distancia (UNED) y el Instituto de Estudios Fiscales de España. 
Finalmente, en el quinto capítulo se verifica la relación entre pobreza y crecimiento económico a través de un modelo econométrico, cuyos resultados arrojan una alta significancia para el crecimiento como factor explicativo (medido por la variación porcentual del producto bruto interno real) y una baja significancia estadística para el gasto social.

\section{LOS ENFOQUES SOBRE LA POBREZA}

Durante siglos, en especial desde el siglo XIX, ha existido una gran preocupación por la pobreza, aun cuando su prioridad en la agenda de la acción política no siempre ha sido manifiesta ni suficiente. La evidencia disponible sugiere que la pobreza es un fenómeno social que involucra múltiples aspectos y, por tanto, las definiciones de la misma y sus causas varían en función de factores como el género, la edad, la cultura y otros múltiples aspectos sociales y económicos. Bajo esta óptica, la definición de la pobreza nos remite a un ámbito subjetivo, donde son pobres las personas que no satisfacen lo que ellas mismas consideran como sus necesidades básicas.

Desde un plano teórico, pese a su relevancia, no existe un modelo para resolver el problema de la pobreza (Verderd, 2007). Por ello, para aproximarnos al estudio de la misma, debemos penetrar en sus causas $y$, a partir de allí, observar cada uno de los diversos enfoques o perspectivas.

\subsection{Enfoque de la teoría del capital social}

Este enfoque propone la falta de activos como causa de la pobreza, encausándose en las nuevas propuestas, que surgen a partir de las críticas a la conceptualización y medición de la pobreza centrada en el ingreso y el consumo (Arriagada et al., 2004). En tal sentido, se sostiene que existen dimensiones que no son fáciles de medir en términos cuantitativos y monetarios, y que influyen fuertemente en la condición de la pobreza. Se trata de variables vinculadas con componentes psicosociales y culturales, y con dimensiones relacionales, normativas, institucionales y cognitivas.

De esta forma, se comprende el capital social como la disponibilidad de los individuos para formar parte de redes de relaciones sociales (asociatividad) y la capacidad específica de movilización de determinados recursos productivos por parte de dichos grupos para su propio beneficio. Desde esta perspectiva, el capital social no es aprovechado eficientemente (y debería serlo), pues conseguiría que un amplio sector de la ciudadanía logre salir de la pobreza. 


\subsection{Enfoque de capacidades y derechos}

Propuesto por Amartya Sen, este enfoque constituye una mirada multidimensional del fenómeno de la pobreza, que agrega el concepto de privaciones. Estas últimas son consideradas como circunstancias insatisfactorias e indeseables, sean materiales, emocionales, físicas o de comportamiento. Sen identifica cinco tipos de derechos que promueven el desarrollo: libertades políticas, servicios económicos, oportunidades sociales, garantías de transparencia y seguridad protectora.

\subsection{Enfoque de exclusión social}

Según el enfoque de exclusión social, ciertas personas están excluidas si no cuentan con una integración social suficiente. Sus principales formas se refieren a situaciones como la no inclusión en sistemas de protección social, la pobreza y la discapacidad, en las que la población no puede participar en actividades comunes y corrientes. También contempla situaciones en las cuales la población es silenciada, ya sea por medio de la estigmatización o la discriminación, lo que equivale a una descalificación social.

\subsection{Enfoque estructural}

Este enfoque considera a la pobreza como derivada de la estructura social de la economía. Por lo general, entiende a la pobreza fundamentalmente como el resultado de los patrones de desigualdad (incluyendo raza, género y desigualdades geográficas), y también las estructuras de poder, económicas, políticas y de elite.

Como se ha podido apreciar, el concepto de pobreza es más complejo de lo que aparenta. Repasar algunos enfoques sobre este fenómeno es esencial para comprenderlo, sin embargo, no es suficiente para enfrentar la pobreza. Por esta razón, en la siguiente sección se repasará un breve marco teórico sobre la política social, para posteriormente enfocar el análisis en el caso peruano.

\section{LA POLÍTICA SOCIAL}

La política social tiene su origen en la necesidad de institucionalizar e instrumentar, como parte de una política de Estado, un conjunto de acciones que permitan a amplios grupos sociales acceder a necesidades que no pueden alcanzar por la vía del mercado, pero que son fundamentales para su subsistencia y desarrollo. 
En sentido amplio, la política social es aquella que tiende a solucionar el problema social de un país, que en términos estrictos se entiende como la existencia de un gran sector de la población en situación de pobreza, y cuyo ejercicio se puede agrupar bajo tres modalidades (Ministerio de Economía y Finanzas MEF, 2006).

- El servicio social, que involucra todas las actividades orientadas a la formación del capital humano a largo plazo, desarrollando en los individuos las capacidades que posibiliten la generación propia de ingresos. Ello implica individuos con mejores condiciones de salud y educación, y un contexto de crecimiento económico que los lleve a integrarse al mercado de trabajo, para de esta manera salir de la pobreza de forma permanente.

- Acciones de previsión social, que procuran proteger a la población ante los riesgos de enfermedades, la vejez y la muerte. Por lo tanto, están orientadas a contrarrestar o mitigar la posibilidad de una interrupción temporal o permanente de la capacidad de un individuo para generar ingresos. Dicha política tiene carácter universal, por lo que el Estado crea instituciones y mecanismos especializados para responder a estos fines.

- Acciones asistenciales, urgentes y de corto plazo, orientadas generalmente a provisionar de medios de subsistencia a la población que vive en condiciones de extrema pobreza o que está impedida de trabajar.

Para que estos objetivos se puedan cumplir, es necesario que primero exista la voluntad política de atender el problema social, el cual, combinado con el conocimiento técnico, permita demostrar su capacidad de gobernar (gobernabilidad) y ejercer un buen gobierno (gobernanza), lo que mejoraría las condiciones de vida de la población. Este elemento es la base esencial que legitima al Estado en una democracia.

El esquema del gráfico 1 resume el lugar que ocupa la política social en el proceso entre la definición del problema social y la acción social, manifestada en el presupuesto de gasto social.

Hoy en día, algunos aspectos de la política social han adquirido un nuevo enfoque o variado ciertos matices. Uno de ellos es el alcance de la intervención del Estado. Ha pasado de la visión clásica, que consideraba que debía financiar, diseñar, proveer, controlar y evaluar la política social, a aceptar e incluso fomentar la participación del sector privado en algunas de estas funciones, antes desarrolladas exclusivamente por el aparato estatal.

Otra visión que se ha transformado es el nivel de responsabilidad del gobierno con la política social, la cual tradicionalmente ha sido atribuida 


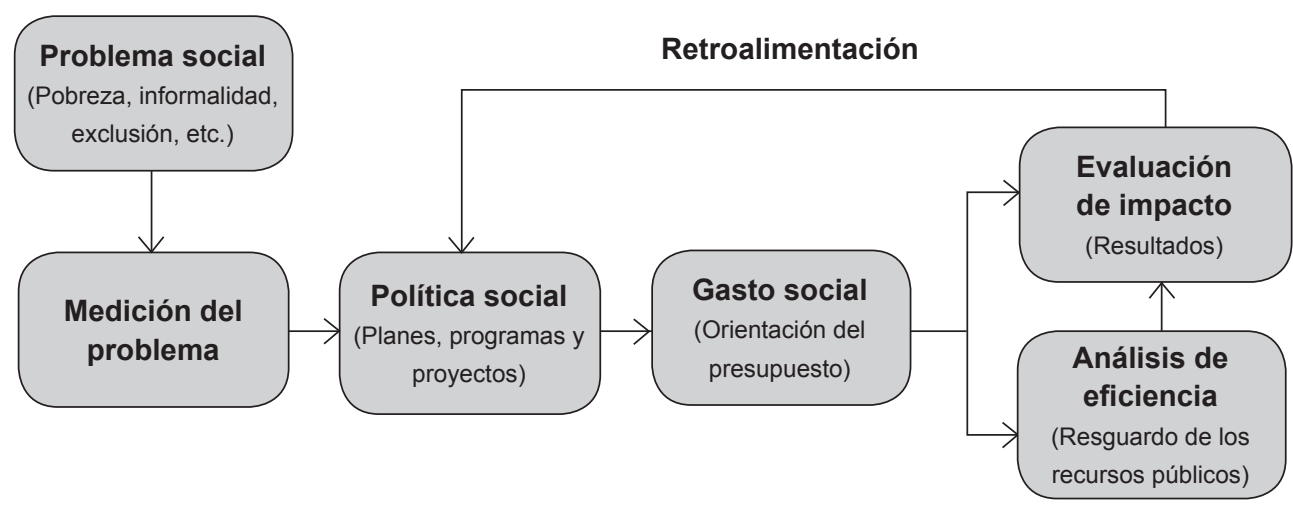

Gráfico 1: Esquema de conexión de la política social Elaboración propia

al gobierno nacional en forma centralizada, regulándola, normándola y financiándola. Sin embargo, actualmente prima un enfoque descentralista, basado en el principio de subsidiariedad, que ha proporcionado mayor responsabilidad a los gobiernos regionales y locales. También se está debatiendo si los subsidios deben ser impulsados por la oferta (supply driven) o por la demanda (demand driven). En el modelo centralista tradicional se enfatiza la primera, pero en el nuevo enfoque existe consenso sobre la mayor eficiencia de los subsidios sobre la demanda.

Finalmente, está la discusión de la universalidad frente a la focalización. En la visión universalista, todos los ciudadanos de una comunidad pueden acceder a los beneficios sociales, sin otro requisito que la ciudadanía y a veces ni siquiera esta es requerida. La visión de focalización está dirigida a determinados subgrupos sociales de ciudadanos desfavorecidos. El peso del debate se acentúa en el costo y la eficiencia de ambas, dado que si bien teóricamente la focalización requiere de menores recursos, también permite elementos de ineficiencia (yuxtaposición de programas, subcobertura y filtración) que pueden hacerla más onerosa con respecto a los resultados. Por otro lado, la política universal puede terminar inflando la necesidad de recursos estatales escasos.

Dirigiendo nuestro enfoque al Perú, durante los últimos 25 años la política social ha aplicado los tres métodos de intervención social de manera conjunta. Sin embargo, cada gobierno ha impreso su propia óptica a cada modalidad, en especial a la intervención a través de acciones asistenciales, tomando en cuenta su propio enfoque sobre el problema de la pobreza y la forma de enfrentarla. 
Al menos hasta los años ochenta, la política social tuvo como paradigma la imagen de un Estado omnipotente que podía actuar como financiador, productor, proveedor y regulador de los servicios básicos de educación, salud y los programas sociales dirigidos a los grupos más vulnerables de la sociedad. De esta manera, al igual que en el resto del continente, después de la Segunda Guerra Mundial las políticas sociales estuvieron influenciadas por el capitalismo de Estado y el crecimiento hacia dentro que propugnaban organismos internacionales, bajo un modelo de búsqueda universalista de la acción social. En suma, la idea prevaleciente era que el Estado estaba en plena capacidad de hacer un uso eficiente y equitativo de los recursos, por ello su asignación se realizaba de manera centralizada, sin tomar en cuenta los intereses, necesidades y características particulares de las poblaciones beneficiadas.

A partir de 1990, con el inicio de las reformas para controlar la hiperinflación y levantar la economía del país, que se tradujo en una política de ajuste severo (política de shock), se elevaron los niveles de pobreza en forma alarmante. En ese contexto, las políticas sociales se transformaron. Se buscó hacerlas más eficientes, junto al acompañamiento que brindaron los organismos multilaterales a los gobiernos de la región, que recomendaban un conjunto amplio de programas especiales, siguiendo criterios de focalización del gasto. Además, muchos de los programas más importantes tuvieron como un componente importante la dimensión participativa, con la cual se buscaba promover el desarrollo del capital social y la sostenibilidad de la inversión.

A inicios del presente siglo, por influencia de los Objetivos de Desarrollo del Milenio (ODM), a los cuales el Perú se adhiere en el año 2000, se inicia lo que podemos considerar un enfoque de capacidades sin un abandono del enfoque de capital social. Durante este periodo se realizó una priorización de los programas considerados de urgencia social, enfatizando el fomento del empleo y la cobertura de servicios básicos. Además, se inicia un sistema de subvenciones directas condicionadas que tuvieron muy buenos resultados, y se reinician los programas de acceso a créditos para vivienda que fueron abandonados en los años ochenta.

En 2006, el segundo gobierno del presidente García planteó un enfoque de capacidades con algunos rasgos del enfoque de inclusión social. En este sentido, se continuó con los programas sociales del gobierno anterior, aunque se aplicaron modificaciones en la gestión, unificando los 82 programas a tan solo 26. A su vez, se aumentó el presupuesto de ciertos programas, debido a la etapa de crecimiento del ciclo de los 
precios de nuestras principales exportaciones, lo que permitió generar los recursos necesarios.

Tras este preámbulo, llegamos a la política social llevada a cabo por el actual gobierno desde julio de 2011. Siguiendo la versión de sus principales representantes, la gestión intenta darle un nuevo enfoque al gasto social, apuntando al problema social de la pobreza ligado a la exclusión social, tomando como base no solo la reducción promedio de la pobreza, sino principalmente la disminución de las disparidades sociales.

El gobierno del presidente Humala, bajo su propuesta de inclusión social, desarrolla la tesis de que las acciones de asistencia no resuelven sosteniblemente la pobreza, ya que no generan cambios en las condiciones que han conducido a los hogares a una situación de precariedad que no pueden alterar mediante sus esfuerzos personales. Por ello, la política se orienta hacia el logro de una situación en la que todas las personas en todo el territorio nacional ejerzan sus derechos, accedan a servicios públicos de calidad y estén en capacidad de aprovechar las oportunidades que catapultan el crecimiento económico, de manera que el origen social o el lugar de nacimiento o residencia no constituyan una traba para la participación en la comunidad nacional, que debe alcanzar condiciones de igualdad.

La inclusión social se orienta, entonces, a la construcción de un marco de políticas públicas dirigidas a cambiar las restricciones, la precariedad y la vulnerabilidad en las que se desenvuelve la vida y la actividad económica de varios sectores de peruanos y peruanas, y que reproducen en ellos, así como en sus entornos y descendientes, condiciones de exclusión. En nuestro país, las enormes disparidades se presentan entre los niveles rural y el urbano, en el Ande y la Amazonía frente a la costa, también entre centros poblados más alejados y ciudades principales, y en otros grupos particularmente vulnerables por razones étnicas, de género, lingüísticas, culturales, de edad, capacidades físicas, entre otras.

\section{GASTO SOCIAL}

En términos presupuestales (ver los gráficos 2 y 3), el gobierno de Fujimori gastó muy poco en el campo social, al menos en la primera parte de su mandato (1990-1995). Se incrementó en su segundo periodo, en particular en los dos últimos años, ad portas del proceso de rereelección de 2000. Como es conocido, dicho gobierno no tenía preparado un plan de emergencia social luego del necesario programa de ajuste (shock), además que muchos de los programas sociales fueron utilizados políticamente. 


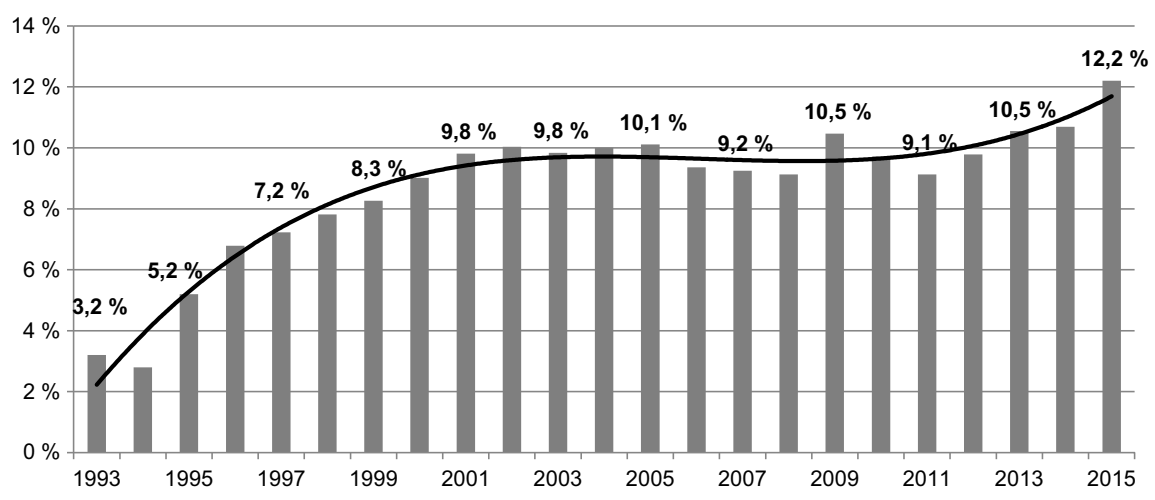

Gráfico 2: Evolución del gasto social total ejecutado como porcentaje del PBI*

* Para los años 2014 y 2015, en base de estimaciónes propias basadas en el primer caso en el Presupuesto Institucional Modificado y en el segundo en el Presupuesto Institucional de Apertura

Fuente: MEF, BCRP

Elaboración propia

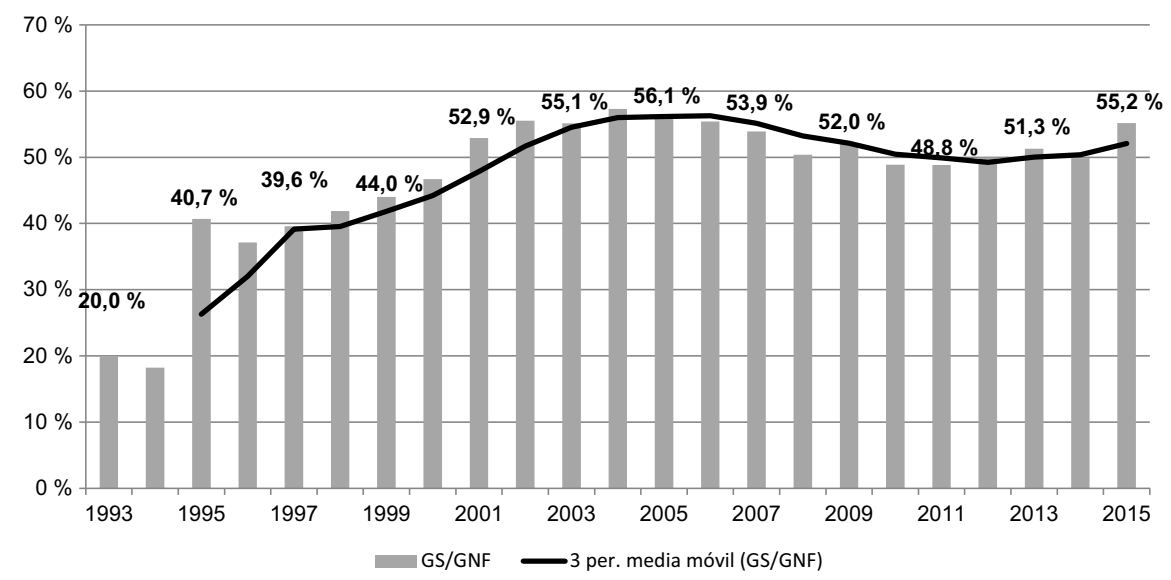

Gráfico 3: Evolución del gasto social total ejecutado como porcentaje del gasto no financiero*

* 2014 (estimación propia) y 2015 (en base del Presupuesto Institucional de Apertura) Fuente: MEF, BCRP

Elaboración propia 
Para ser justos, debe señalarse que los ingresos fiscales eran muy inferiores a los de la siguiente década y que existía otro problema social con un impacto similar a la pobreza: el terrorismo.

Por su parte, el gobierno de Alejandro Toledo fue el que ejecutó un mayor gasto social. Esta evaluación es más contundente si la realizamos en términos del gasto no financiero, lo cual ocurrió cuando el boom económico experimentado por el país aún no se iniciaba. No obstante-como se ha anotado antes-, la pobreza se mantuvo, por lo que podría calificarse como una política de gasto social poco eficiente.

Aunque el gobierno de Alan García ejecuto un nivel de gasto social ligeramente inferior que el de Toledo -solo afectado por la reducción en 2008, producto de la crisis económica mundial iniciada ese año-, queda la sensación de que debió haber sido superior, teniendo en cuenta que fue el gobierno que contó con mayor cantidad de recursos fiscales en varias décadas.

Finalmente, el gobierno actual, presidido por Ollanta Humala, viene ejecutando un gasto social que en términos del PBI es superior al de Toledo y García, con una tendencia creciente para finales de este mandato, aunque en términos del gasto no financiero es inferior a los gobiernos mencionados. Sin embargo, el gasto social básico (educación, salud y saneamiento) muestra un crecimiento mayor, el cual no se presentó en ninguno de los gobiernos anteriores, hecho que resulta coherente con la política de desarrollo e inclusión social.

\subsection{La política de gasto social del gobierno de Ollanta Humala}

El gobierno actual ha centralizado la política social. El propósito es realizar una gestión por resultados, colocando la máxima atención en el ciclo de vida de la persona, desde el periodo de gestación hasta la ancianidad. A su vez, emplea mecanismos de evaluación más transparentes. Este es un cambio muy importante, encaminado en la dirección positiva. Incorpora un esquema de metas y tiene indicadores ligados al presupuesto que permiten monitorear los resultados, sobre todo de los grupos más vulnerables. Cabe mencionar que en el presupuesto 2014 se involucró aproximadamente el $60 \%$ de los recursos dentro del esquema presupuestario por resultados y el $90 \%$ de los recursos de los programas sociales del Ministerio de Desarrollo e Inclusión Social (Midis). 
En términos operativos, el enfoque de la actual política de desarrollo e inclusión, según el plan inicial trazado por la ministra del Midis, Carolina Trivelli y su equipo, se basa en un modelo que abarca tres horizontes temporales complementarios: corto, mediano y largo plazo (ver el gráfico 4).

\begin{tabular}{|c|c|c|}
\hline \multirow{5}{*}{$\begin{array}{l}\text { Alivio temporal } \\
\text { - Población en pobreza extrema } \\
\text { (transferencias) } \\
\text { - Condiciones básicas de vida } \\
\text { (vivienda, abrigo, alimentación) } \\
\text { - Tercera edad }\end{array}$} & \multirow{3}{*}{$\begin{array}{l}\text { Desarrollo de capacidades } \\
\text { - Desarrollo de capacidades } \\
\text { (trabajo, innovación, gestión y } \\
\text { organización) } \\
\text { - Acceso a servicios (infraestruc- } \\
\text { tura básica, inclusión financiera) }\end{array}$} & $\begin{array}{l}\text { Generación de oportunidades } \\
\text { para la siguiente generación } \\
\text { - Salud básica y nutrición } \\
\text { - Educación básica }\end{array}$ \\
\hline & & \\
\hline & & crónica de menores de 5 años \\
\hline & Mediano plazo & $\begin{array}{l}\text { (Organización Mundial de la } \\
\text { Salud) }\end{array}$ \\
\hline & $\begin{array}{l}\text { - Incrementar hogares } \\
\text { con acceso a servicios }\end{array}$ & $\begin{array}{l}\text { - Disminuir la inasistencia de } \\
\text { niños de entre } 3 \text { y } 5 \text { años }\end{array}$ \\
\hline Corto plazo & $\begin{array}{l}\text { (electricidad, agua, desagüe } \\
\text { y telefonía) }\end{array}$ & $\begin{array}{l}\text { de edad a la educación } \\
\text { básica regular }\end{array}$ \\
\hline & - Ingreso autónomo & \\
\hline
\end{tabular}

Seguimiento y evaluación por resultados

Gráfico 4: Horizontes temporales de intervención

Fuente: Midis (Dirección general de políticas y estrategias)

Elaboración: Midis

En el corto plazo, el esfuerzo está centrado en el alivio temporal de los hogares a través de la asistencia directa. En el mediano plazo, el énfasis se coloca en el desarrollo de capacidades orientadas a mejorar el acceso de los hogares a servicios e infraestructura básica y a incrementar su autonomía en cuanto a la generación de ingresos y procesos de inclusión financiera. Para el largo plazo, las intervenciones están orientadas a la generación de oportunidades para la siguiente generación, con énfasis en la protección y mejora del capital humano: nutrición, salud y educación de calidad.

Asimismo, este análisis incluye el importante rol del hogar, en tanto es considerado como el espacio en el que se expresan las diversas etapas del ciclo de vida y el receptor natural de las intervenciones del Estado. Es precisamente bajo el enfoque de ciclo de vida (ver el gráfico 5) donde se definen los cinco ejes del Plan Estratégico Incluir para Crecer: nutrición infantil, desarrollo infantil temprano, desarrollo integral de la niñez y la adolescencia, inclusión económica y protección del adulto mayor. 
EE1. Nutrición infantil

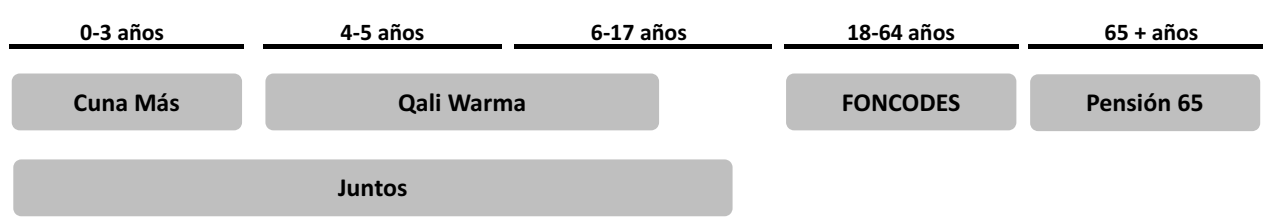

Gráfico 5: Programas sociales del Midis

Fuente: Midis (Dirección general de Políticas y Estrategias)

Elaboración: Midis

Bajo este enfoque, el Estado genera las condiciones para que los hogares como conjunto mejoren su situación actual y de forma sostenible en el futuro, y puedan así enfrentar las necesidades del ciclo vital de sus integrantes a través de la combinación de medidas. En tal sentido, cinco programas sociales canalizan los recursos del Estado a cargo del Midis y atienden a los hogares en situación de pobreza y pobreza extrema en los diferentes momentos de su ciclo vital, colaborando con los ejes estratégicos de la política de inclusión y llevando a la práctica el modelo de política social que combina acciones de alivio y de desarrollo.

Cabe recalcar que, acorde con la nueva política, entre 2011 y 2015, el actual gobierno incrementó en $84,4 \%$ el gasto social básico y en 98,7 \% el gasto social complementario, tal como se puede ver en el cuadro 1 , con una tasa promedio de crecimiento de $13,8 \%$ en el gasto social total.

En la tabla anterior destaca el avance en dos ámbitos esenciales: educación y salud. Con respecto al primero, se aprecia que la ejecución durante 2013 creció, en términos reales, en $28,2 \%$ con respecto a lo ejecutado en 2012, sustentado por el direccionamiento de mayores recursos en los rubros educación inicial y primaria (incremento de 29,1 \% y 20,8 $\%$, respectivamente). De otra parte, los recursos destinados a la función salud aumentaron en 9,0 \% en términos reales, acentuándose los recursos destinados a salud individual, tanto por el gran volumen de los mismos como por su dinámica.

Trasladándonos a los resultados, solo podemos examinar preliminarmente algunos indicadores, dado que este gobierno aún no concluye y la evaluación de impacto debe realizarse posteriormente. Sin embargo, podemos analizar tendencias y comparar el avance a la fecha con respecto 
Cuadro 1

Gasto público y social según grandes componentes ${ }^{1}$ (en millones de Nuevos soles)

\begin{tabular}{|l|c|c|r|r|r|}
\hline & \multicolumn{5}{|c|}{ Presupuesto ejecutado } \\
\hline & 2011 & 2012 & \multicolumn{1}{|c|}{2013} & \multicolumn{1}{|c|}{$2014^{7}$} & $2015^{7,8}$ \\
\hline Gasto no financiero del gob. general $^{2}$ & 87864 & 99462 & 112500 & 123546 & 130621 \\
Gasto social total & 42897 & 49716 & 57691 & 61596 & 72042 \\
1. Gasto social no previsional $^{3}$ & 31258 & 38105 & 44833 & 48321 & 60081 \\
1.1 Gasto social básico $^{4}$ & 14211 & 16713 & 19811 & 21640 & 26209 \\
1.2 Gasto social complementario $^{5}$ & 17047 & 21393 & 25022 & 26681 & 33872 \\
2. Gastos previsionales $^{6}$ & 11639 & 11611 & 12858 & 13275 & 11962 \\
\hline
\end{tabular}

1 Considera una taxonomía del gasto social basada en registros típicos del clasificador funcional programático.

2 Considera el gasto de los tres niveles de gobierno: nacional, regional y local.

3 Incluye gasto de tipo administrativo.

4 En el marco del Consenso de Oslo, este se define como los gastos en: educación básica (inicial y primaria), salud básica, alimentación y nutrición, y agua y saneamiento.

5 Corresponde al conjunto de actividades y/o proyectos de carácter social que no están considerados dentro del gasto social básico, como, por ejemplo: educación secundaria, educación superior, infraestructura social y productiva, electrificación rural, carreteras rurales, etc.

6 Considera el gasto previsional de tipo administrativo.

7 Estimado en base a cifras presupuestales.

8 En base del Presupuesto Institucional de Apertura (PIA).

Fuente: Sistema Integrado de Administración Financiero (SIAF)

a las metas establecidas por esta administración a 2016, entre ellas, las ligadas al problema principal de la pobreza, como reducir la brecha de pobreza ${ }^{3}$ para alcanzar el $6 \%$. Esta meta ya se cumplió, situándose en $5,8 \%$ en 2014. También se propuso lograr que solo el $20 \%$ de la población se encuentre por debajo del umbral monetario de pobreza ${ }^{4}$ y la pobreza extrema alcance el $5 \%$. En el primer indicador se llegó al 22,7 \% hasta 2014, y parece ser una meta difícil de alcanzar, dados los bajos niveles de crecimiento económico. Respecto de la meta de pobreza extrema de $5 \%$ para 2016, ya fue superada, pues en 2014 esta se ubicó en 4,3 \%. Finalmente está la cifra de $7 \%$ a 2016 establecida para la pobreza extrema,

3 Proporción del gasto que le falta a un pobre para cubrir el costo de la canasta básica de consumo o alcanzar la línea de la pobreza.

4 Medido por la incidencia de la pobreza monetaria (personas que residen en hogares cuyo gasto per cápita es insuficiente para adquirir una canasta básica de alimentos y no alimentos: vivienda, vestido, educación, salud, transporte, etc.), y que representa la proporción de pobres o de pobres extremos como porcentaje del total de la población. 
medida con el ingreso autónomo -es decir, ingreso neto de transferencias y donaciones públicas-. Aunque al momento de preparar este artículo no se han publicado cifras para 2014, en 2013 se llegó al 7,4%. Es razonable esperar que estas metas sean alcanzadas, aún con las perspectivas de un crecimiento cercano al 2,5\% del PBI en 2015.

En el cuadro 2 se aprecia que los niveles de pobreza para el periodo 2011-2014 solo se han reducido en 5,1 puntos porcentuales, comparados con el 18,4 \% de reducción de 2006 a 2010. Sin embargo, debe tenerse en cuenta el menor crecimiento del PBI, que como veremos más adelante es el elemento central para la reducción de la pobreza, y al hecho de que a medida que nos acercamos al núcleo duro de la pobreza (donde ocurren las disparidades), se vuelve más difícil alcanzar grandes niveles de reducción.

Por ello, es imprescindible destacar la reducción de los índices de pobreza extrema en el área rural y en la sierra rural (ámbitos de mayor pobreza), que muestran el énfasis del actual gobierno en la reducción de disparidades. En el gráfico 6 se observa una reducción de la pobreza extrema de 2,0 puntos porcentuales entre 2011 y 2014, de la pobreza rural de 10,1 \% y en la sierra rural de 11,9 \%. Además, en la población pobre con lengua nativa ${ }^{5}$, la pobreza se ha reducido en 9 \% entre 2011 y 2014.

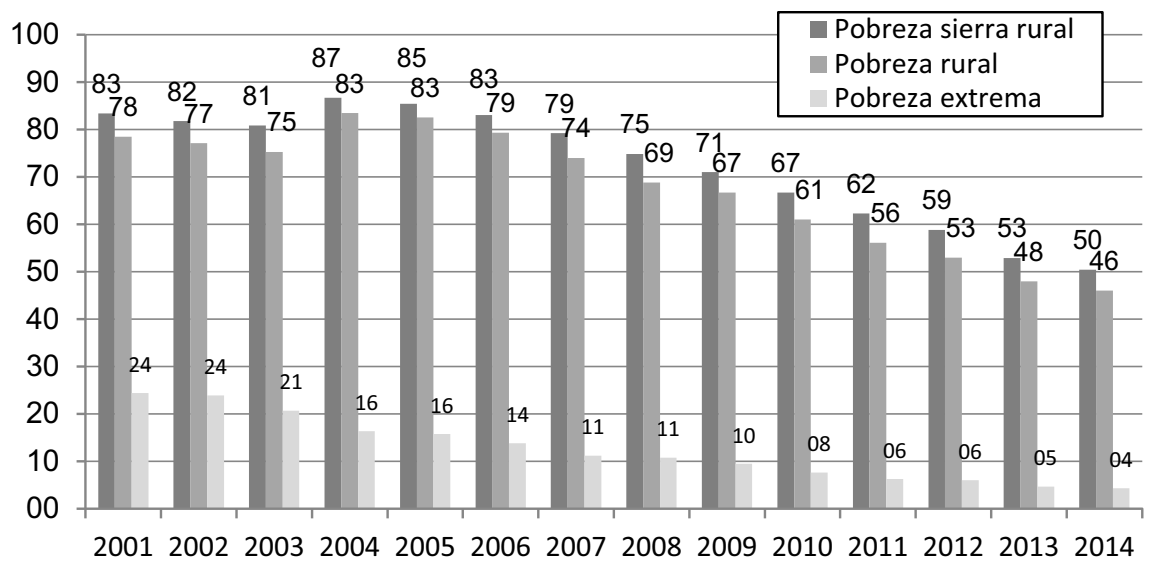

Gráfico 6: Evolución de la incidencia de la pobreza monetaria 2001-2014

Fuente: INEI

Elaboración propia

5 Que incluye quechua, aimara y otras lenguas nativas. 


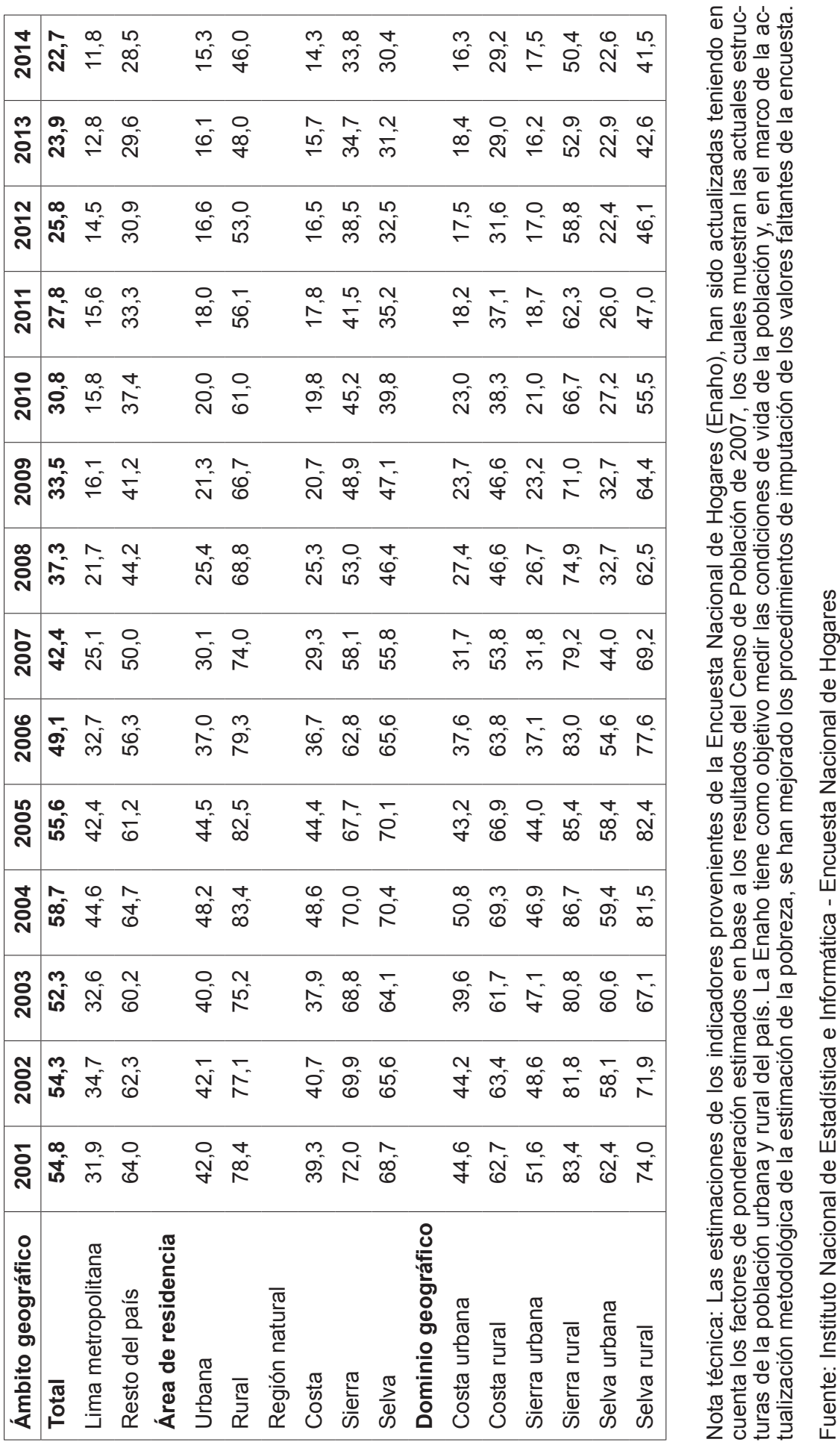


Por otra parte, tomando en cuenta la pobreza multidimensional, aun cuando en el gráfico 7 se aprecia que la tasa neta de asistencia escolar nacional en el nivel inicial para niños de 3 a 5 años aumentó solo 4,8 \%, tasa inferior a los $6,4 \%$ de aumento de los tres primeros años del gobierno anterior, para los próximos años se estiman alzas notables, debido al crecimiento del presupuesto en educación. Sin embargo, lo más saltante es que la asistencia en la población pobre aumenta en el periodo 2011-2014 en $12,4 \%$, al pasar de $58,5 \%$ a $70,9 \%$. Aunque aún no hay cifras oficiales para el año 2014, lo mismo se observa en la población rural, cuya tasa aumenta en 11,3\% entre 2011 y 2013, y en el ámbito geográfico de la sierra, donde aumenta en 10,2\% en el mismo periodo. Además, todos estos indicadores muestran progresos superiores a los alcanzados en el periodo 2006-2008, al inicio del anterior gobierno.

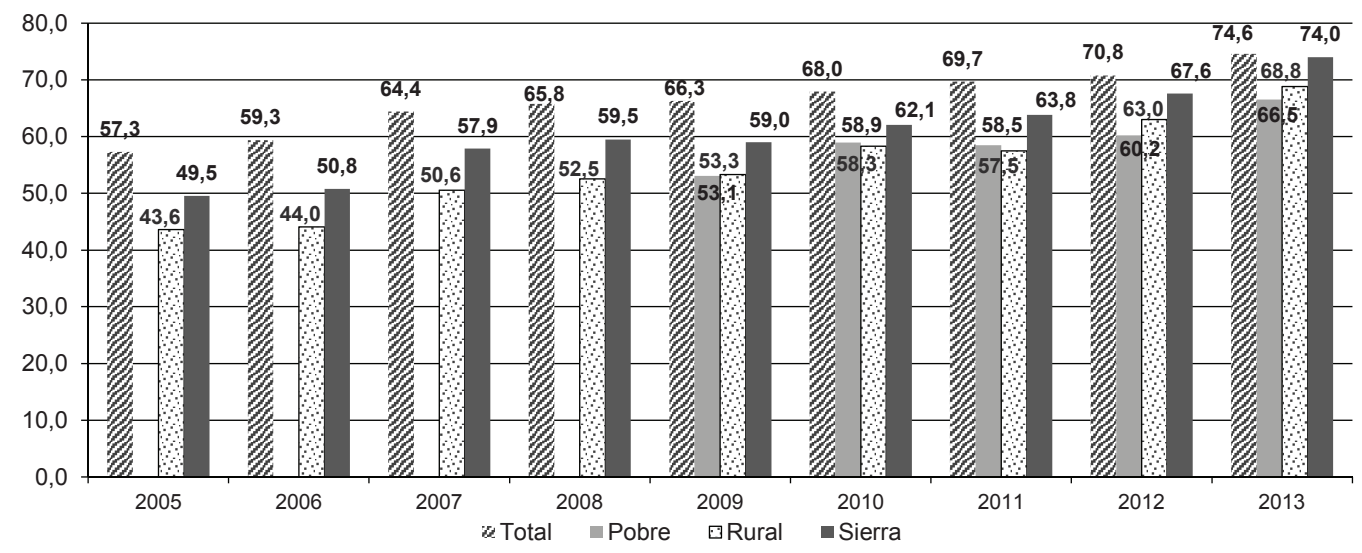

Gráfico 7: Tasa neta de asistencia a educación inicial (de 3 a 5 años)

Fuente: Enaho-INEI

Elaboración propia

De igual forma, en el gráfico 8, basado en cifras publicadas por el Instituto Nacional de Estadística e Informática (INEI), según la encuesta nacional demográfica de salud familiar (Endes) se puede observar que la desnutrición crónica infantil (DCI), otro indicador de gran importancia, muestra una evolución favorable. Se redujo en 5,4\% en el periodo 2011-2014, pasando de 19,5\% en 2011 a 14,1 \% en el primer semestre de 2014. Sin embargo, para alcanzar la meta de desnutrición crónica infantil establecida por el gobierno para el año 2016, se requiere una disminución mínima de 2,5 puntos porcentuales anuales. 


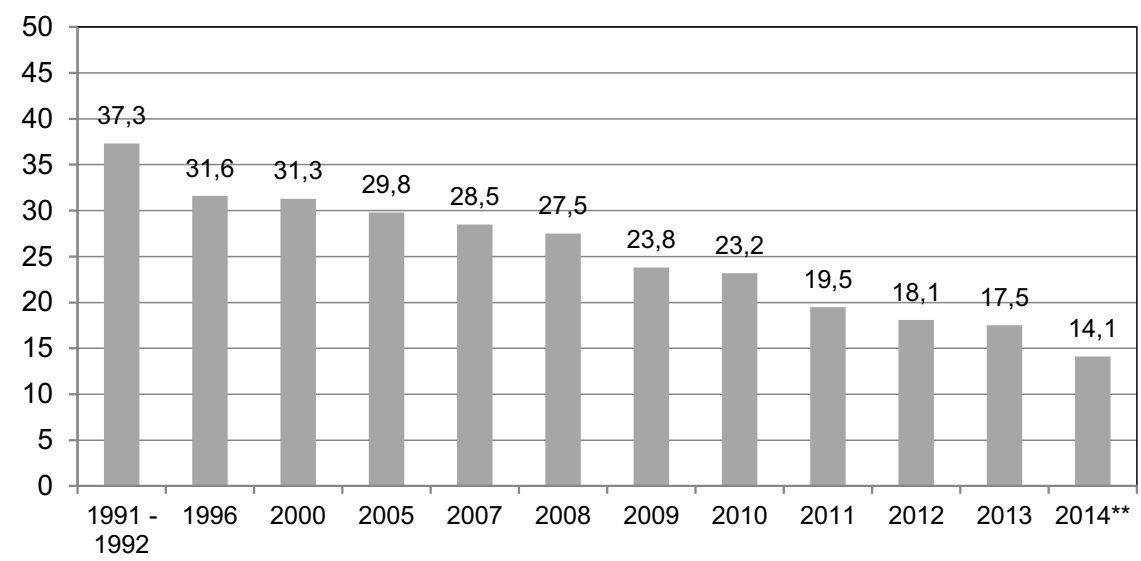

Gráfico 8: Tasa de desnutrición crónica infantil* (niños menores de 5 años)

* Patrón Organización Mundial de la Salud (OMS).

** Preliminar al $1 .{ }^{\text {er }}$ semestre.

Fuente: INEI - ENDES

Elaboración propia

Sin embargo, en la población infantil rural el panorama no es tan alentador. Luego de una reducción de 5,1 puntos porcentuales entre los años 2011 y 2012, pasando de $37 \%$ a 31,9 \% en 2013, la tasa de desnutrición crónica infantil en el sector rural creció ligeramente, hasta el 32,3\%. Aunque, según cifras de resultados preliminares de la Endes 2014, al primer semestre la DCI rural se redujo a $28,2 \%$; sin embargo, persisten situaciones como la del departamento de Huancavelica (el más pobre del país hasta 2014), donde aún supera el $42 \%$. En el mismo sentido, otro indicador relacionado, la anemia en niños entre seis meses y tres años de edad, pasó de $41,6 \%$ en 2011 a 46,4 \% en 2013, y en el primer semestre de 2014 aumentó a $50 \%$.

En cuanto al acceso a agua por red pública, como se aprecia en el gráfico 9, dicho indicador ha mostrado un incremento sostenido desde el año 2004. No obstante, el progreso entre 2011 y 2013 ha sido muy amplio, alcanzando los 6 puntos porcentuales, muy superior al $1 \%$ del periodo 2006-2008. Lo más interesante es que dicha variación ha favorecido especialmente al sector rural, donde ha mostrado un aumento de $18,2 \%$ entre 2011 y 2013, y de 18,1 \% en la población pobre, igualmente superior al decrecimiento estadístico de 2,2 \% en el periodo 2006-2008. 


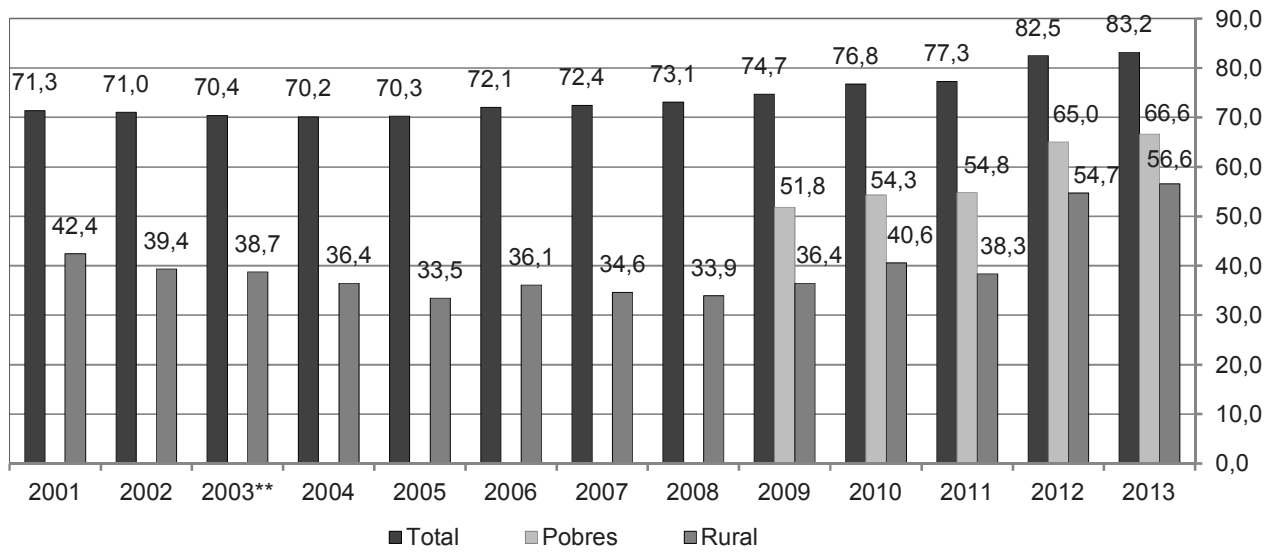

Gráfico 9: Población con acceso de agua por red pública. 2001-2013* (porcentaje)

* Abastecimiento de agua: incluye agua de red pública dentro de la vivienda, red publica fuera de la vivienda pero dentro del edificio y pilón de uso público.

** Para el año 2003, el periodo de encuesta está referido al año comprendido entre mayo 2003 y abril 2004.

Fuente: Enaho-INEI

Elaboración propia

Finalmente, como se puede apreciar en el gráfico 10, los hogares que acceden al servicio de red pública de energía eléctrica han mejorado en el total nacional en 2,4 \% entre 2011 y 2013, y en el ámbito rural aumentó en 7,4\% en similar periodo. Sin embargo, resultan cifras inferiores al $4,5 \%$ y $10,5 \%$ alcanzado entre 2006 y 2008 , aunque entre los pobres aumentó en 7,8\% de 2011 a 2014.

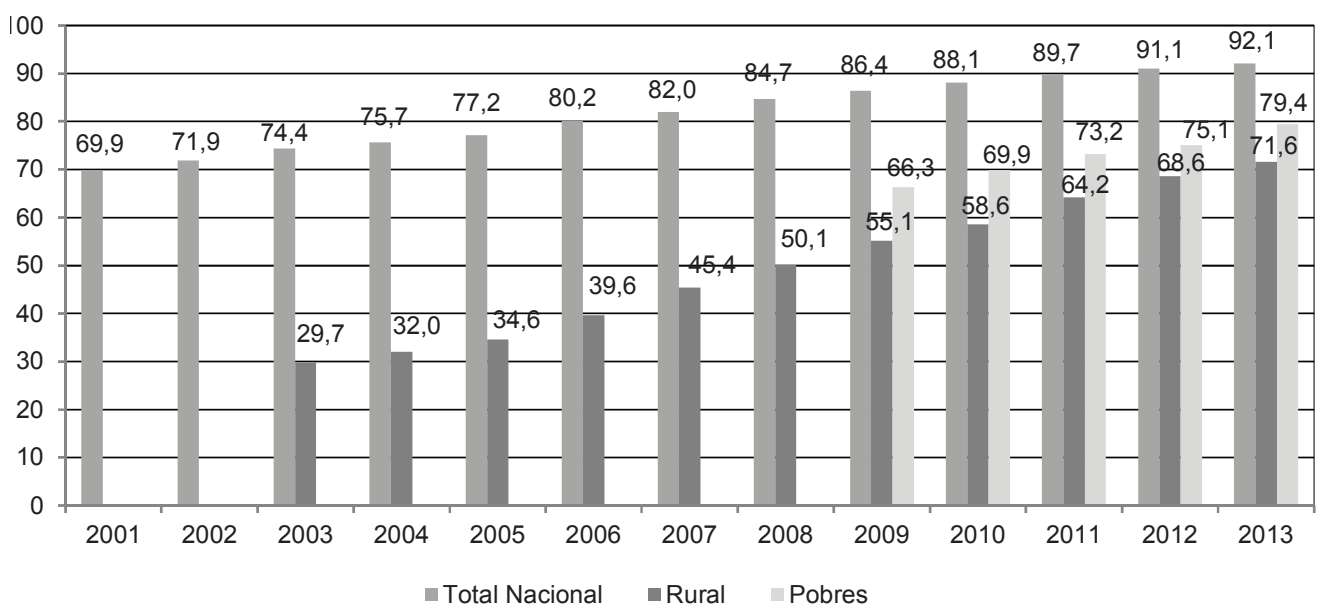

Gráfico 10: Hogares que acceden al servicio de energía eléctrica por red pública (porcentaje) Fuente: Enaho-INEI

Elaboración propia 
De los resultados preliminares observados, podemos ver una tendencia alentadora con respecto a las metas trazadas por el propio gobierno, previo reconocimiento de que atravesamos por una nueva etapa de reducción del ciclo de crecimiento de nuestra economía.

\section{POBREZA Y CRECIMIENTO ECONÓMICO}

En general, como bien anotan todos los especialistas en la materia y ha reafirmado el Banco Mundial ${ }^{6}$, el componente más relevante de la reducción de la pobreza en el Perú y el resto de América Latina durante los últimos 10 años es la mayor dinámica del mercado laboral originada por el crecimiento económico. La diferencia correspondería a las políticas fiscales de redistribución.

A decir del renombrado economista peruano Javier Iguiñiz, "en la pobreza influyen el crecimiento, la política social y el gasto social de lucha contra la pobreza", pero "el crecimiento explica las decenas, la política social las unidades, y el gasto de lucha los decimales" (Chacaltana, 2006). En este sentido, debemos dimensionar adecuadamente las políticas sociales, ya que si bien son necesarias, para lograr una reducción sustancial de la pobreza tiene que existir crecimiento, fenómeno que en la década anterior provino en su mayor parte de una coyuntura externa favorable.

Para el caso peruano, entre 1995 y 2013 se puede estimar que existe una alta correlación entre los puntos porcentuales de reducción de la pobreza y el crecimiento económico medido por la variación porcentual del PBI real (ver el gráfico 11), con un coeficiente de correlación de Pearson de 0,653, significativo al $99 \%$, y un coeficiente de determinación de $42,35 \%$.

Otro ejercicio de prueba estándar en esta línea es el modelamiento de la pobreza a partir de datos trasversales o de panel utilizando modelos de variable dependiente binaria (logit y probit). El mismo se relaciona a las características de los grupos de individuos a partir de los datos de las encuestas de hogares, con variables como el nivel educativo del jefe de familia, la edad de los padres, el tamaño del hogar, la zona de residencia, la lengua, el gasto, el empleo, el nivel salarial, la recepción de transferencias y donaciones, la atención de salud, entre otros. Sin embargo, como el propósito de este ejercicio es demostrar la relación entre el crecimiento y la pobreza, hemos optado por un modelo de series de tiempo, asumiendo

6 Ver noticia en http://gestion.pe/opinion/editorial-gestion-gasto-imperceptible2090820 . 
las dificultades que la falta de datos presenta en un modelo de este tipo, pero reconstruyendo a juicio experto la información necesaria a partir de diversas fuentes.

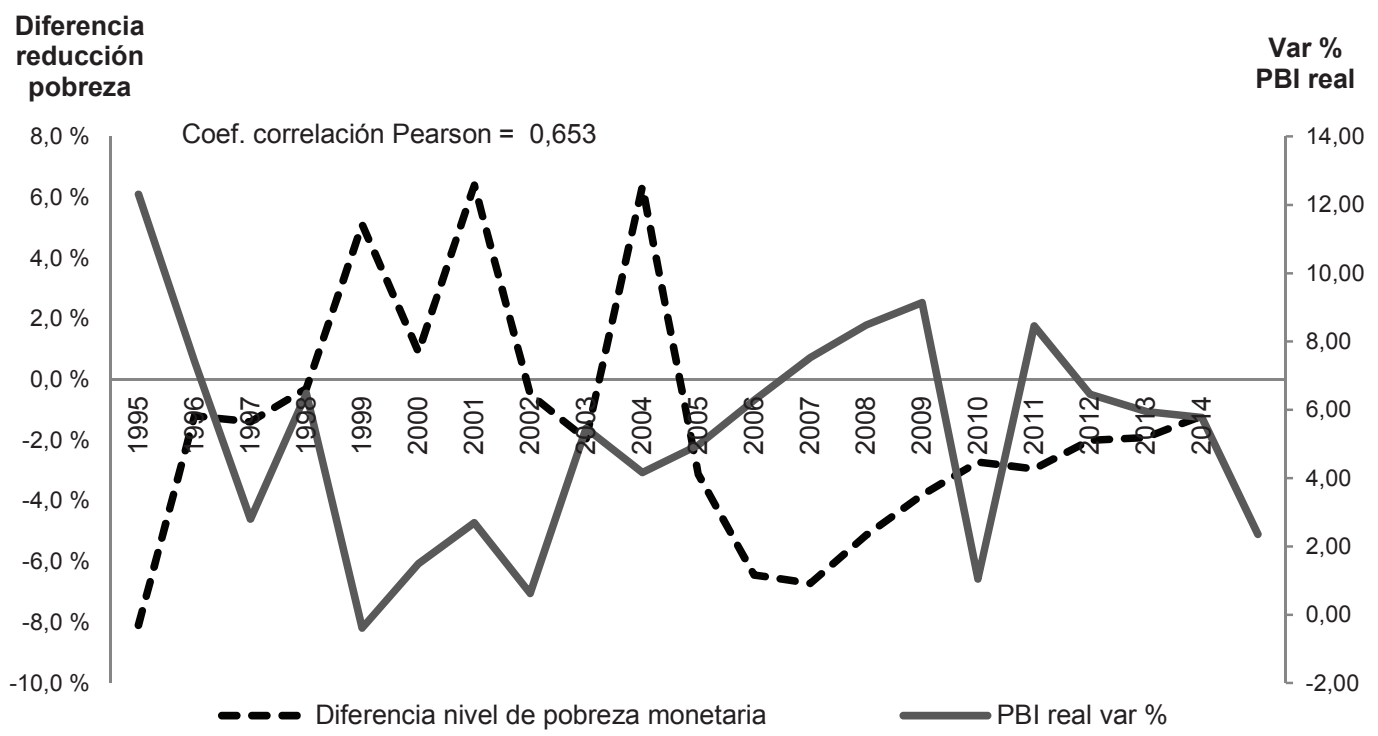

Gráfico 11: VAR \% PBI real versus puntos porcentuales de reducción de pobreza Elaboración propia

Este modelamiento temporal de la pobreza se efectúa con información que va de los años 1990 a 2014, usando como variable dependiente el indicador de pobreza monetaria (POBR) en diferencias y como regresores el porcentaje del gasto social respecto al PBI (GSPBI), como una medida del esfuerzo dedicado por el gobierno a la política social, la variación porcentual del PBI real (Varpbi), el índice de Gini (GINI) rezagado tres periodos, y una variable dummy que refleje la diferente eficacia del gasto en épocas cercanas a las elecciones regionales y presidenciales.

Como resultado (ver el cuadro 3) encontramos que la variación porcentual del PBI real y el índice de Gini resultan significativas. Por su parte, el gasto social no muestra mayor significancia estadística, sin embargo, los signos sí son los adecuados y todas las pruebas relacionadas al modelo son consistentes ${ }^{7}$. Como se puede observar, un punto porcentual de

7 El ajuste global del modelo es bueno. Lo validan las pruebas de significancia estadística, como el de normalidad de los residuos (Jarque-Bera), el RESET de Ramsey (LM Test), la correlación serial (Bruesch-Pagan-Godfrey) y la heterocedasticidad 
incremento en el PBI real podría generar una reducción de 0,74 puntos porcentuales en la pobreza.

Cuadro 3

Variable dependiente: $\mathrm{D}(\mathrm{POBR})$

Método: Mínimos cuadrados

Fecha: 02/24/16 Tiempo: 14:52

Muestra (ajustada): 1994-2014

Observaciones incluidas: 21 después de ajustes

\begin{tabular}{lclrl}
\hline Variable & Coeficiente & Error est. & Estático-t & Prob. \\
\hline C & 0,029635 & 0,017825 & 1,662523 & 0,1159 \\
D(GS_PBI) & $-0,143260$ & 0,009243 & $-1,550013$ & 0,1407 \\
VARPBI & $-0,748527$ & 0,245886 & $-3,044202$ & 0,0077 \\
D(GINI(-3)) & 0,700001 & 0,22911 & 3,055305 & 0,0076 \\
DUM3 & $-0,007265$ & 0,016441 & $-0,441899$ & 0,6645 \\
\hline R-cuadrado & 0,565024 & Media var. dependiente & $-0,014905$ \\
R-cuadrado ajustado & 0,45628 & Desv. est. var. dependiente & 0,038622 \\
Error est. de regresión & 0,028479 & Akaike criterio info & $-4,075032$ \\
Suma cuadrados residuos & 0,012977 & Schwarz criterio & $-3,826337$ \\
Probabilidad Log & 47,78784 & Hannan-Quinn criterio & $-4,021059$ \\
Estadístico-F & 5,195911 & Durbin-Watson estad. & 1,840568 \\
Prob(Estadístico-F) & 0,007074 & & \\
\hline
\end{tabular}

Es necesario anotar, como bien ha señalado el Banco Mundial ${ }^{8}$, que probablemente el crecimiento económico por sí solo ya no sea un elemento suficiente para sacar a los pobres crónicos de la pobreza, pero que conjuntamente con otras políticas públicas de desarrollo y asistencia social bien diseñadas, sí puede conseguirse.

\section{REFLEXIONES FINALES}

En los últimos 25 años se ha venido dando un aumento sostenido del presupuesto dedicado al gasto social, desde un $6,5 \%$ de la década de los no-

(Breusch-Godfrey). Todos ellos muestran que estadísticamente la variable de gasto social no es significativa, sin embargo, prevalece en este caso, atendiendo a la teoría económica.

8 Ver Vakis, R., Rigolini, J. y Lucchetti, L. (2015). Los olvidados: pobreza crónica en América Latina y el Caribe. Washington. Banco Mundial. 
venta hasta un 10,2 \%, medido sobre el PBI en el último quinquenio. Esto significa que todos los gobiernos se dan cuenta de que solucionar el problema social adquiere cada vez mayor importancia, como un elemento de cohesión social y estabilidad democrática.

El actual gobierno ha definido su política social en términos de la inclusión, tomando como base no solo la reducción promedio de la pobreza, sino principalmente la reducción de las disparidades sociales. Ello implicó atacar también la desigualdad como factor que contribuye a perennizar la pobreza y que se manifiesta en las enormes disparidades entre el campo y la ciudad, en la sierra y la selva con respecto a la costa, etc. Ello, por supuesto, ha demandado un cambio en el análisis de las causas de la pobreza, y transitar hacia una política de ciclo de vida que pueda trasversalmente atacar dichas disparidades.

Aún no se puede afirmar que este nuevo enfoque ha mejorado la eficiencia y eficacia del gasto social en el Perú. Es muy probable que una futura evaluación de impacto sobre los promedios generales arroje resultados inferiores o al menos no concluyentes sobre su eficacia y eficiencia, pero es imprescindible no quedarse en dichos promedios y observar más detenidamente los resultados en los grupos que son el objetivo del modelo. Los resultados preliminares en este sentido son aún ambiguos, aunque alentadores con respecto a las metas trazadas por la actual política social, reconociendo que atravesamos una nueva etapa del ciclo de crecimiento de nuestra economía.

Finalmente, sobre este mismo aspecto de la eficacia del gasto social, debe señalarse que -tal como todos los expertos coinciden- a medida que nos acercamos al núcleo duro de la pobreza, se vuelve más difícil alcanzar grandes niveles de reducción. Por otra parte, el ciclo favorable externo parece estar entrando a un punto de inflexión y, como se demostró, este es un factor clave para mantener el ritmo hacia los objetivos propuestos. En este sentido, antes de caer en la "trampa de los países con ingreso medio", el gobierno debe corregir algunos aspectos, basados en sus propias metas, pero continuar concentrándose en lo esencial de su nueva política, es decir, la exclusión social, manifestada por las disparidades existentes, incluso cuando ello tenga un alto costo, al menos desde un punto de vista político. 


\section{REFERENCIAS BIBLIOGRÁFICAS}

Abrahamson, P. (1996). Social Exclusion in Europe: Old Wine in New Bottles. Conferencia de la European Science Foundation (ESF), Blarney, 26-30 de marzo.

Alcazar, Lorena. (2010). Agenda Pendiente de Reformas en el Perú. Proyecto Advocating for Economic Reform in Peru. Lima. Confiep, Instituto Apoyo y Cipe.

Arriagada, I., Miranda, F. y Pávez, P. (2004). Lineamientos de acción para el diseño de programas de superación de la pobreza desde el enfoque del capital social. Guía conceptual y metodológica. Santiago de Chile: Cepal.

Atria, R. (2006). Políticas Sociales: Concepto y Diseño. Un Marco De Referencia. (Documento de trabajo n. ${ }^{\circ}$ ). Santiago de Chile: Instituto de Asuntos Públicos. Universidad de Chile.

Banco Mundial. (2005). Informe de Pobreza del Perú: Oportunidades para todos. Washington: Departamento de Reducción de Pobreza y Administración Económica Región de América Latina y el Caribe. Report n. ${ }^{\circ}$ 29825-PE.

Bardález, C. (2002). Marco social multianual 2010-2012. Lima: Informe preliminar del capítulo de Salud para el para el MSM.

Cepal. (2013). Panorama Social de América Latina. (Documento Informativo). Santiago de Chile: Copyright.

Chávez, G. (2008). Criterios para la Asignación del Gasto Social en Programas de Salud y Nutrición en el Perú. Lima: CIES.

Chacaltana, Juan. (2006). ¿Se puede prevenir la Pobreza? Informe Final. Lima: CIES.

Dubois, F., Chavez, J. y Cusato, A. (2004). Programas sociales, salud y educación en el Perú: Un balance de las políticas sociales. Lima: Instituto Peruano de Economía Social de Mercado-IPESM. IPE.

Escobal, J., Saavedra, J. y Torero, M. (1999). Los Activos de los Pobres en el Perú. (Documento de Trabajo R-361). Washington: BID.

Estrada, H. y Perea, A. (2008). Los programas Sociales en el Perú 1990-2007: Del alivio a la superación de la pobreza. Informe de coyuntura n. ${ }^{\circ}$ 026/20072008. Lima: Unidad de análisis del departamento de comisiones-Congreso de La Republica del Perú. 
Figueroa, A., Altamirano, T. y Sulmont, D. (1996). Exclusión social y desigualdad en el Perú. Lima: OIT. 151 páginas.

Figueroa, G. (2014). Exclusión Social en el Perú: Hacia una nueva política social. Lima: INEI.

Fiszbein, A. y Schady, N. (2009). Panorama general-Transferencias monetarias condicionadas: Reduciendo la pobreza actual y futura. Banco Internacional de Reconstrucción y Fomento. Banco Mundial.

INEI. (2014). Evolución de la Pobreza Monetaria 2009-2013. Informe Técnico.

Jaramillo, M. y Sánchez, A. (2011). Impacto del programa Juntos sobre nutrición temprana. Lima: Grade. Documento de Investigación n. 61.

O’Brien, E. (2001). Informe sobre programas sociales. Mesa de Concertación de Lucha Contra la Pobreza.

Martínez, R., y Collinao, M. (2010). Gasto social: modelo de medición y análisis para América Latina y el Caribe. Santiago de Chile: Cepal.

Martínez, R., Palma, A., Flores, L. y Collinao, M. (2012). El impacto económico de las politicas sociales. Santiago de Chile: Cepal.

Ministerio de Economía y Finanzas. (2006). El Gasto Público Social en el Perú: Taxonomía, definiciones y opciones de política. (Documento de trabajo). Lima: Dirección de Estudios Macro Sociales-MEF.

Ministerio de Economía y Finanzas. (2011). Programación Presupuestaria por Resultados: Una evaluación del Programa Articulado Nutricional (PAN). Lima: Dirección General de Presupuesto Público.

Ministerio de Economía y Finanzas. (2012). Presupuesto del Sector Público del año 2013: Guía de Orientación. Lima: Dirección General de Presupuesto Público.

Ministerio de Economía y Finanzas. (2013). Ley de Presupuesto 2014: Guía de Orientación al Ciudadano. Lima.

Ministerio de Economía y Finanzas. (2013). Reporte Seguimiento del Presupuesto: Al mes de diciembre 2013. Lima: Dirección General de Presupuesto Público.

Ministerio de Economía y Finanzas. (2014). Reporte Seguimiento del Presupuesto: Al mes de marzo 2014. Lima: Dirección General de Presupuesto Público. 
Mendoza W., Leyva J. y Flor J. (2012). La distribución del ingreso en el Perú: 1980-2010. Lima: Departamento de Economía-Pontificia Universidad Católica del Perú (PUCP).

Ministerio de Desarrollo e Inclusión Social. (2012). Conocer para incluir: Lineamientos para el seguimiento, evaluación y gestión de la evidencia del Midis. Lima.

Ministerio de Desarrollo e Inclusión Social. (2012). Una política para el desarrollo y la inclusión social en el Perú. Lima.

Ministerio de Desarrollo e Inclusión Social. (2012). Lineamientos para la gestión articulada intersectorial e intergubernamental orientada a reducir la desnutrición crónica infantil en el marco de las politicas de desarrollo e inclusión social. Lima.

Ministerio de Desarrollo e Inclusión Social. (2013). Estrategia Nacional de Desarrollo e Inclusión Social-Incluir para Crecer (2. edición). Lima.

Ministerio de Educación. (2004). Cifras de la Educación 1998-2003. Lima: Unidad de Estadística Educativa.

Ministerio de Educación. (2013). Memoria Institucional 2012-2013. 1. ${ }^{a}$ edición. Lima.

Ministerio de Salud. (2011). Situación Actual del Sistema de Salud en el Perú: Políticas de Salud Nacionales y regionales. Presentación. Lima.

Ministerio de Salud. (2011). Plan Estratégico Sectorial Multianual 2012-2016. Propuesta Preliminar. Lima.

Monge, A. y Winkelried, D. (2010). Dinámica en la demanda por programas sociales en el Perú. Lima: CIES.

Olivares, A. (2007). Perú: Notas sobre las cuentas del sector social. Presentación. Lima: MEF.

Øyen, E. (2000). Social capital formation as a poverty reduction strategy. Ginebra: Unesco/Most and CROP/ISSC Symposium, UN World Summit for Social Development.

Paugam, S. (1993). La Disqualification Sociale: Essai sur la Nouvelle Pauvreté. París: PUF.

Parodi, T. (2005). Economía de las Políticas Sociales. Lima: Centro de Investigación de la Universidad del Pacífico. Biblioteca Universitaria. 
Pascó-Font, A. y Saavedra, J. (2001). Reformas estructurales y bienestar: una mirada al Perú de los noventa. Lima: Grade.

Putnam, R. (1993). Making Democracy Work: Civic Traditions In Modern Italy. Princeton: Princeton University Press.

Ruiz, R. (2010). Sobre la evolución del gasto público social en América Latina y su papel para la estabilización económica. Santiago de Chile: Cepal.

Saavedra, J. y Pascó-Font, A. (2001). Reformas estructurales y bienestar. Lima: Grupo de Análisis para el Desarrollo.

Sen, A. (2000). Desarrollo y libertad. Lima: Editorial Planeta.

Soto, H. y Schuschny, A. (2009). Diseño de indicadores compuestos de desarrollo sostenible. Guía metodológica. Santiago de Chile: Cepal.

Subirats, S. (2004). Pobreza y exclusión social: Un análisis de la realidad española y europea. Colección Estudios Sociales n. 16. Barcelona: Fundación "la Caixa".

Tanaka. M. y Trivelli, C. (2002). Las trampas de la focalización y la participación. Pobreza y políticas sociales en el Perú durante la década de Fujimori. Lima: Instituto de Estudios Peruanos (IEP).

Usaid Perú. (2013). Análisis del avance en la ejecución del presupuesto público en salud 2013. Lima.

Valderrama, J. y Pichihua, T. (2011). Propuestas metodológicas para la focalización individual de los programas sociales. Lima: BCRP.

Vakis, R., Rigolini, J. \& Lucchetti, L. (2015): Los olvidados: pobreza crónica en América Latina y el Caribe. Washington: Banco Mundial.

Vásquez, E. y Franco, M. (2003). Fusión de programas sociales en el Perú: Un fondo de inclusión social como propuesta. (Documento de discusión DD/07/03). Lima: Centro de Investigación de la Universidad del Pacífico.

Vásquez, E. (2007). La brecha entre el discurso político y la gestión pública a favor de los más pobres: un balance de los primeros meses del segundo gobierno de Alan García. Lima: Centro de Investigación de la Universidad del Pacífico. Documento de Investigación Economía y Sociedad n. ${ }^{\circ}$ 64-CIES.

Vásquez, E. (2013). Las políticas y programas sociales del gobierno de Ollanta Humala desde la perspectiva de la pobreza multidimensional. (Documento 
de discusión DD/13/07). Lima: Centro de Investigación de la Universidad del Pacífico.

Verdera, F. (2007). La pobreza en el Perú: Un análisis de sus causas y de las políticas para enfrentarla. Serie Análisis Económico n.․ 24. Lima: IEP.

Webb, R. (2012). Pobreza y dispersión poblacional. Informe Final. Lima: CIES-USMP.

Yamada, G., Castro J., y Bacigalupo, J. (2012). Desigualdad monetaria en un contexto de rápido crecimiento económico: El caso reciente del Perú. Revista Estudios Económicos, 24, 65-77. Lima: BCRP. 